\title{
Leaky-mode expansion of the electromagnetic field inside dispersive spherical cavity
}

P. Jakobsen, M. Mansuripur, and M. Kolesik

Citation: Journal of Mathematical Physics 59, 033501 (2018); doi: 10.1063/1.5006956

View online: https://doi.org/10.1063/1.5006956

View Table of Contents: http://aip.scitation.org/toc/jmp/59/3

Published by the American Institute of Physics

\section{Articles you may be interested in}

Generalized delta functions and their use in quantum optics

Journal of Mathematical Physics 59, 012102 (2018); 10.1063/1.4985938

Topological edge states for disordered bosonic systems

Journal of Mathematical Physics 59, 031901 (2018); 10.1063/1.5002094

Reduction by invariants and projection of linear representations of Lie algebras applied to the construction of nonlinear realizations

Journal of Mathematical Physics 59, 033502 (2018); 10.1063/1.4989890

$(1+1)$ Newton-Hooke group for the simple and damped harmonic oscillator

Journal of Mathematical Physics 59, 032901 (2018); 10.1063/1.4986383

Exact analytical solutions of the Schrödinger equation for a two dimensional purely sextic doublewell potential

Journal of Mathematical Physics 59, 032101 (2018); 10.1063/1.4997532

Singularities of the transmission coefficient and anomalous scattering by a dielectric slab Journal of Mathematical Physics 59, 033507 (2018); 10.1063/1.5027195

\section{PHYSICS TODAY}

WHITEPAPERS
MANAGER'S GUIDE

Accelerate R\&D with

Multiphysics Simulation
READ NOW

PRESENTED BY

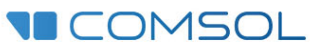




\title{
Leaky-mode expansion of the electromagnetic field inside dispersive spherical cavity
}

\author{
P. Jakobsen, ${ }^{1}$ M. Mansuripur, ${ }^{2}$ and M. Kolesik ${ }^{2}$ \\ ${ }^{1}$ Department of Mathematics and Statistics, University of Troms $\phi$, Troms $\phi$, Norway \\ ${ }^{2}$ College of Optical Sciences, University of Arizona, 1630 East University Boulevard, \\ Tucson, Arizona 85721, USA
}

(Received 28 September 2017; accepted 14 February 2018; published online 1 March 2018)

\begin{abstract}
Rigorous justification is presented for a recently introduced method to construct leakymode expansions of electromagnetic fields excited inside a spherical cavity filled with a dispersive, lossy medium. In a departure from the traditional approaches, our construction does not rely on Green's functions, rather it starts from a judiciously chosen auxiliary meromorphic function. Convergence of both the series expansions and of the over-completeness relations for the leaky modes is proven for a realistic model of chromatic dispersion. Published by AIP Publishing. https://doi.org/10.1063/1.5006956
\end{abstract}

\section{INTRODUCTION}

Leaky or quasi-normal electromagnetic modes occur in a number of practically important circumstances. While the standard description in terms of normal modes is only admissible for closed systems, quasi-normal modes, similar to their quantum-mechanical counterparts (see, e.g., Ref. 1), offer a natural framework for open and lossy waveguides and optical cavities. ${ }^{2}$

Not surprisingly, numerous applications span different fields including the description of optical waveguides, ${ }^{3}$ resonator cavities, ${ }^{4,5}$ photonic structures,,${ }^{6,7}$ plasmons,,${ }^{8,9}$ and even black holes, ${ }^{10}$ offering a simplified and more economical alternative to the treatment based on a continuum of normal modes.

The interest of the research community concentrates on both the practical computational issues ${ }^{11-13}$ and on the better understanding of the general properties and the mathematics underlying the description of physical systems using quasi-normal modes ${ }^{14-19}$ in various geometries. ${ }^{20-22}$

However, despite the long history of leaky modes and non-Hermitian quantum mechanics ${ }^{23}$ and electromagnetics, the proper theory and even the physical concepts are still somewhat obscure (see, e.g., Ref. 24). The most fundamental question of how much of the physics can be captured by the leaky modes (e.g., Refs. 12, 19, and 25) has not yet been answered in the most general terms. There are also some technical issues that are subject to debate and different treatments. For instance, various ways of mode-volume calculation ${ }^{26}$ and quasi-normalization ${ }^{27,28}$ have been described and contrasted in the literature, oftentimes re-inventing methods established previously or in different fields.

This work concentrates on the fundamental question of completeness, ${ }^{29,30}$ i.e., on when and how the set of leaky electromagnetic modes can be used as a basis to compute arbitrary electromagnetic fields. Typically, rigorous studies of open and non-Hermitian systems have been done on a case-by-case basis. Our work deals with the spherical optical cavity formed by a homogeneous dispersive and lossy medium. Needless to say, this is, from a practical standpoint, a highly relevant system. In our previous study, ${ }^{31,32}$ we concentrated on its physical properties, such as the relation between the cavity Q-factors and the properties of the medium. The goal of this work is to provide a rigorous proof that the system of outgoing resonant modes is indeed (over-)complete and also to give mathematical justification to a novel method to construct resonant-mode expansions of arbitrary functions.

The question of completeness for optical cavity modes has been posed and addressed before. Notably, the studies by Leung et al. establish the quasi-normal modes as complete in several specific systems, such as a one-dimensional (Fabry-Pérot-like) cavity ${ }^{30}$ and also spherical cavity filled with dispersive and lossy medium. ${ }^{29}$ 
There are several novel aspects presented in this work. Regarding the physical description of the cavity medium, ours is the first exact treatment that admits a fully realistic dispersive/lossy material. Some of the previous description either neglected the loss while capturing the chromatic dispersion, ${ }^{33}$ giving rise to what is a causality-violating approximation. Leung et al. ${ }^{29}$ treated cavities with both chromatic dispersion and losses, but their approach relied on a frequency-independent background refractive index, which was needed to carry out the completeness proof. We are utilizing a proper Lorentz oscillator description (see also Ref. 24 for a 1D system), which has an important general property that for very high frequency the refractive index converges to unity. As a result, waves with increasingly high-frequencies eventually cease to "see" the cavity, and this has consequences regarding the details of the completeness proof.

Another departure from the route taken by the previous studies lies in the method used to construct the leaky-mode expansion. While in our preceding work ${ }^{32}$ we demonstrated the practicalities of the new approach, including examples of leaky-mode expansions in different cavity-geometries, ${ }^{31}$ here we present a rigorous mathematical justification. An issue which, to the best of our knowledge, has not been previously discussed in detail is that any Lorentz medium will give rise to multiple accumulation points ${ }^{24}$ for the resonant energies in the complex plane and that these must be excised from the region enclosed in the integration contour used to derive the discrete representation of the electromagnetic field.

The main conceptual difference form the standard approach to the completeness problem ${ }^{29,30}$ is that we analyze the convergence of the series directly, without invoking any properties of Green's functions. ${ }^{28,34}$ The advantage of the present method is that it is more general. Specifically, we do not need to assume that the function to be represented as a resonant-state expansion is a product of time-evolution in the system (i.e., optical cavity in the present case or a Hamiltonian in the quantum case) with modified, outgoing boundary conditions. We simply assume that the expanded function resulted from an excitation in the original physical system, without modification of its properties. As an additional benefit, our analysis of the rate of convergence of the leaky-mode series allows us to understand why such expansions exhibit rather slow convergence, despite the fact that already a few-term approximation is often extremely good. Our method can also be straightforwardly modified in order to obtain various over-completeness relations.

Our paper is organized as follows. Electromagnetic modes of a dielectric spherical cavity, expressed in terms of spherical harmonics, are reviewed next. In Sec. III, we indicate how the continuum of real-frequency modal fields give rise to discrete sets of leaky modes with complex-valued frequencies. We outline our method for construction of a leaky-mode expansion of electromagnetic fields excited inside the sphere in Sec. IV. In Sec. V, we first prove that the leaky-mode expansion converges, provided that an auxiliary function that embodies the non-uniqueness of the series is chosen appropriately. Then we go on to show that the convergence is indeed to the desired target function. Our method naturally leads to over-completeness relations, and these are discussed in Sec. VI. We summarize and offer some concluding remarks in Sec. VII.

\section{ELECTROMAGNETIC MODES OF SPHERICAL CAVITIES}

Consider a dielectric sphere of radius $a$ placed in a vacuum, assuming that the relative dielectric permittivity $\epsilon(\omega)$ can be described by a single Lorentz oscillator. Such a medium is conventionally parameterized by giving its resonant angular frequency $\omega_{r}$ and the corresponding damping constant $\gamma$, while the strength of the oscillator can be expressed through the plasma frequency $\omega_{p l}$. With such a notation, the frequency-dependent susceptibility of the medium is given as

$$
\chi(\omega)=\frac{\omega_{p l}^{2}}{\omega_{r}^{2}-\omega^{2}-i \gamma \omega} .
$$

Throughout this work, we assume that the chromatic dispersion inside the sphere can be approximated by a single term of this form. While realistic material models often require several Lorentz oscillators to approximate their frequency-dependent properties, our restriction to a single-oscillator medium suffices to illustrate the issues that chromatic dispersion brings into considerations regarding the convergence of leaky-mode expansions. It will become obvious in what follows that additional Lorentz 
oscillators bring nothing qualitatively new for the convergence of the resonant series because each of multiple Lorentz-oscillator singularities (described next) can be investigated the same way. For the same reason, we will only consider non-magnetic media.

Rather than susceptibility, most of the time we utilize the refractive index, $n^{2}=\epsilon=1+\chi$, written as

$$
n(\omega) \equiv \sqrt{1+\chi(\omega)}=\sqrt{\frac{\left(\omega-\omega_{1}\right)\left(\omega-\omega_{2}\right)}{\left(\omega-\omega_{3}\right)\left(\omega-\omega_{4}\right)}},
$$

where

$$
\omega_{1,2}= \pm \sqrt{\omega_{r}^{2}+\omega_{p l}^{2}-\gamma^{2} / 4}-i \gamma / 2 \text { and } \omega_{3,4}= \pm \sqrt{\omega_{r}^{2}-\gamma^{2} / 4}-i \gamma / 2
$$

In realistic situations, we have $\gamma \ll \omega_{r}$, and all $\omega_{i}$ lie in the lower complex half-plane, as indicated in Fig. 1. Representation (2) of the refractive index is convenient for our purposes because it reveals analytic properties important for the leaky modes. In particular, the refractive index exhibits branch cut points at $\omega_{1,2}$ and two susceptibility poles at $\omega_{3,4}$.

The electromagnetic field oscillating with a given angular frequency $\omega$ can be decomposed into eigenmodes in terms of spherical Bessel functions. We use the following to denote vector spherical harmonics

$$
\mathbf{Y}_{l m}=Y_{l m} \vec{r}, \quad \boldsymbol{\Psi}_{l m}=r \nabla Y_{l m}, \quad \boldsymbol{\Phi}_{l m}=\vec{r} \times \nabla Y_{l m}
$$

which simplify the notation for the vector modes of the spherical cavity. Assuming a fixed real-valued frequency and given angular-momentum numbers $l, m$, transverse-electric (TE) modes can be written as

$$
\begin{aligned}
& \vec{E}=E(r) \boldsymbol{\Phi}_{l m}, \\
& \vec{B}=B^{(1)}(r) \boldsymbol{\Psi}_{l m}+B^{(r)}(r) \mathbf{Y}_{l m},
\end{aligned}
$$

while transverse magnetic (TM) modes are parameterized like

$$
\begin{aligned}
& \vec{B}=B(r) \boldsymbol{\Phi}_{l m}, \\
& \vec{E}=E^{(1)}(r) \boldsymbol{\Psi}_{l m}+E^{(r)}(r) \mathbf{Y}_{l m} .
\end{aligned}
$$

For these modes to satisfy the Maxwell equations, functions $E(r), B(r)$ must obey radial Bessel equations and must be continuous at the sphere surface. In the TE case, also the radial derivative must be smooth, i.e.,

$$
E\left(a^{-}\right)=E\left(a^{+}\right) \text {and } E^{\prime}\left(a^{-}\right)=E^{\prime}\left(a^{+}\right)
$$

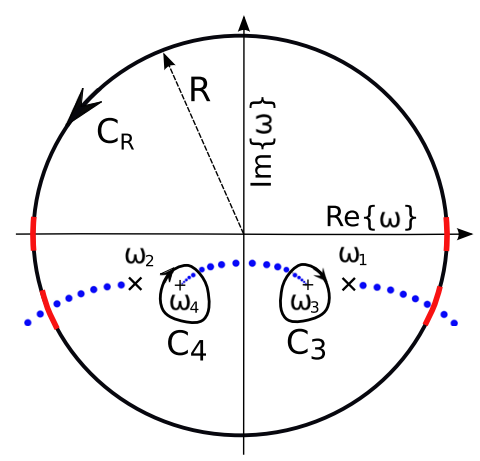

FIG. 1. Frequency-dependent refractive index has two branch points $\omega_{1,2}$ and two susceptibility poles $\omega_{3,4}$, both pairs located below the real axis. Resonant frequencies of leaky modes are marked by full circles (blue), and $\omega_{3,4}$ and $\infty$ are their accumulation points. Thick full lines, with components $C_{R}, C_{4}$, and $C_{3}$, represent the integration contour used to derive our resonant-mode expansion. Contour components $C_{3,4}$ together with the highlighted sections of $C_{R}$ require special treatment because they must pass in the vicinity of some poles (see text). 
while in the TM case the Maxwell equations imply the following boundary conditions:

$$
B\left(a^{-}\right)=B\left(a^{+}\right) \text {and }\left.\left(\frac{d}{d r}+\frac{1}{r}\right) \frac{B(r)}{\epsilon(\omega)}\right|_{r=a^{-}}=\left.\left(\frac{d}{d r}+\frac{1}{r}\right) \frac{B(r)}{\epsilon(\omega)}\right|_{r=a^{+}} .
$$

Having specified $E(r), B(r)$, one can calculate the rest of the modal functions.

\section{A. Modal functions-TE case}

A convenient representation of the real-frequency radial function of a TE mode can be chosen as follows:

$$
\begin{aligned}
& E(r)=\frac{1}{\left.N \sqrt{D^{+} D^{-}}\right]} j_{l}\left(k_{i} r\right), \quad r<a, \\
& E(r)=\frac{i}{N}\left[\sqrt{\frac{D^{-}}{D^{+}}} h_{l}^{(1)}\left(k_{o} r\right)-\sqrt{\frac{D^{+}}{D^{-}}} h_{l}^{(2)}\left(k_{o} r\right)\right], \quad r>a,
\end{aligned}
$$

where we use spherical Bessel and Hankel functions and where $D^{ \pm}(\omega)$ are frequency-dependent coefficients which ensure that proper boundary conditions are satisfied at the sphere interface. $N$ is an unimportant normalization factor, and

$$
k_{i}(\omega)=\frac{\omega n(\omega)}{c}, \quad k_{o}(\omega)=\frac{\omega}{c}
$$

are wavenumbers inside and outside of the sphere, respectively. Conditions (9) lead to

$$
\begin{aligned}
& D^{+}=\frac{\left(k_{o} a\right)^{2}}{2}\left[n(\omega) j_{l+1}\left(k_{i} a\right) h_{l}^{(1)}\left(k_{o} a\right)-j_{l}\left(k_{i} a\right) h_{l+1}^{(1)}\left(k_{o} a\right)\right], \\
& D^{-}=\frac{\left(k_{o} a\right)^{2}}{2}\left[n(\omega) j_{l+1}\left(k_{i} a\right) h_{l}^{(2)}\left(k_{o} a\right)-j_{l}\left(k_{i} a\right) h_{l+1}^{(2)}\left(k_{o} a\right)\right] .
\end{aligned}
$$

\section{B. Modal functions-TM case}

The real-frequency radial function can be parameterized in a similar way,

$$
\begin{aligned}
& B(r)=\frac{1}{\left.N \sqrt{M^{+} M^{-}}\right]} j_{l}\left(k_{i} r\right), \quad r<a, \\
& B(r)=\frac{i}{N}\left[\sqrt{\frac{M^{-}}{M^{+}}} h^{(1)}\left(k_{o} r\right)-\sqrt{\frac{M^{+}}{M^{-}}} h^{(2)}\left(k_{o} r\right)\right], \quad r>a,
\end{aligned}
$$

where $M^{ \pm}$play the same role as $D^{ \pm}$. Boundary conditions (10) result in

$$
M^{+}=\frac{\left(k_{o} a\right)^{2}}{2 \epsilon}\left[\epsilon j_{l}\left(k_{i} a\right) h^{\prime(1)}\left(k_{o} a\right)-\frac{k_{i}}{k_{o}} j_{l}^{\prime}\left(k_{i} a\right) h^{(1)}\left(k_{o} a\right)-\frac{(1-\epsilon)}{k_{o} a} j_{l}\left(k_{i} a\right) h^{(1)}\left(k_{o} a\right)\right],
$$

and the expression for $M^{-}$is obtained by exchanging $h^{(1)}\left(k_{o} a\right) \leftrightarrow h^{(2)}\left(k_{o} a\right)$. Having specified explicit expressions for the radial modal functions $E(r)$ and $B(r)$ for any real frequency $\omega$ allows us, in principle, to obtain the expansion of an arbitrary time-dependent radiative electromagnetic field inside and outside of the spherical cavity. Such an expansion is discrete in $l, m$ but is continuous in $\omega$.

The central question concerning the leaky modes is if it is possible to replace the continuum expansion with a discrete one, albeit with some limitations. Specific to this work is if such an expansion can be possible for a realistic medium with chromatic dispersion.

\section{LEAKY MODES IN DISPERSIVE SPHERICAL CAVITIES}

With the leaky-mode expansion of $B(r)$ being technically similar to that of $E(r)$, we shall restrict our attention to the case of TE electromagnetic fields. 
The description of the real-frequency cavity fields given in Sec. II suggests an intuitive way to obtain the leaky modes. Far from the origin, spherical Hankel functions $h^{1,2}$ that appear in the modal field represent outgoing and incoming waves. It is possible to eliminate the incoming waves if one finds a complex-valued frequency at which $D^{+}$vanishes.

Thus, the leaky modes are identified with the complex-valued solutions of

$$
D^{+}(z)=0 \text { and } D^{-}(z)=0,
$$

where the first and second options lead to outgoing and incoming modes, respectively.

For $D^{+}(z)=0$, the incoming wave in the TE modal field vanishes, but the modal field needs to be normalized differently. One possibility is as follows (for $\omega=\omega_{q}$ ):

$$
\begin{array}{ll}
E(r)=g_{<}(\omega) \equiv \omega h_{l}^{(1)}\left(k_{o} a\right) j_{l}\left(k_{i} r\right), & r<a, \\
E(r)=g_{>}(\omega) \equiv \omega j_{l}\left(k_{i} a\right) h_{l}^{(1)}\left(k_{o} r\right), & r>a .
\end{array}
$$

This function is obviously continuous across the sphere surface, and it is easy to check that it has a continuous derivative when $D^{+}\left(\omega=\omega_{q}\right)=0$. Thus, it is a valid solution with a complex-valued angular frequency.

For a dispersive medium under consideration, there are infinitely many solutions of $D^{+}\left(\omega_{q}\right)=0$, giving rise to an infinite family of outgoing leaky modes. The question is in what sense can such a set be utilized as a basis in the space of physically allowable electromagnetic fields. Properties of the resonance frequencies $\omega_{q}$ are crucial for understanding such questions.

Figure 1 shows a schematic illustration of their locations. There are four families, each associated with a feature of the dispersive refractive index. The first infinite sequence of leaky-mode frequencies spans the region of low frequencies and accumulates in the vicinity of $\omega_{3}$ where the index of refraction diverges; there are infinite number of solutions inside the arbitrary small neighborhood of this point. The second family is also infinite and populates a curve between $\omega_{4}$ and $\infty$, the latter being an accumulation point of this sequence. Because of the symmetry, every pole $\omega_{q}$ comes with its counterpart $-\omega_{q}^{*}$. There is therefore a third family of poles which has an accumulation point at $\omega_{4}$, and the fourth family starts at $\omega_{2}$ and approaches infinity.

\section{CONSTRUCTION OF LEAKY-MODE EXPANSION}

The traditional point of departure for investigations into normal-mode expansions is Green's function with the outgoing radiative boundary conditions. We proceed differently and construct a leaky-mode representation of physically admissible fields inside the cavity with the help of a judiciously chosen auxiliary meromorphic function.

Our method highlights two important aspects of such an expansion, namely, certain degrees of freedom or non-uniqueness and a design approach informed by the requirement of the convergence for the resulting functional series.

Let us imagine that an electromagnetic field was excited within a spherical cavity by illuminating it from the outside. According to Sec. II, at time $t$, a component of the field with a fixed angularmomentum numbers $l, m$ can be expanded into real-frequency modes like so

$$
E^{l, m}(r, t) \sim \int A_{l, m}\left(\omega_{0}\right) j_{l}\left(k_{i}\left(\omega_{0}\right) r\right) e^{-i \omega_{0} t} d \omega_{0},
$$

where $A_{l, m}(\omega)$ is the angular spectral amplitude of the field. If one could replace the spherical Bessel function $j_{l}\left(k_{i}\left(\omega_{0}\right) r\right)$ with a discrete series running over complex $\omega_{q}$, integration over $\omega_{0}$ would give us a discrete-sum representation of this electric field.

Before addressing issues concerning the convergence of such a series in a rigorous way, let us first outline our approach in broad strokes. Consider a function of a complex variable $\omega$,

$$
G(\omega)=U(\omega) \frac{\exp \left[i k_{o}(\omega) a\right] j_{l}\left(k_{i}(\omega) r\right)}{\left(\omega_{0}-\omega\right) D^{+}(\omega)},
$$

where $U(\omega)$ is some meromorphic function to be fixed later. We want to utilize the degree of freedom $U$ provides, but it must not have poles except at $\omega=0$. We shall assume that its behavior at small 
$\omega$ is such that function $G(\omega)$ remains regular at the origin. This requirement allows for a pole of a degree less than the order $l$ of the spherical Bessel function that appears in $G$. To derive a discretesum expression for a target function, we calculate a contour integral (see Fig. 1) along a contour $C=C_{R}+C_{3}+C_{4}$ consisting of a big circle, $C_{R}=|\omega|=R$, and two small circles $C_{3,4}$ of radius $R_{3,4} \rightarrow 0$ excising respective neighborhoods of $\omega_{3,4}$. The latter contour components are necessary because $\omega_{3,4}$ are accumulation points for poles of $G$, and the excised portion of the complex plane, no matter how small, contains an infinite number of poles. Without removing these portions, we could not apply the residue theorem. Assuming that the integrand given by $G(\omega)$ decays sufficiently fast as $R$ growths and that the integral along the contours encircling $\omega_{3,4}$ also vanishes in the limit $R_{3,4} \rightarrow 0$,

$$
\int_{C} G(\omega)=0
$$

one can apply the residue theorem to obtain a discrete sum.

Figure 1 highlights certain sections of the integration contour that require closer attention and analysis. Planning to return to this later, for the moment we assume that their contribution vanishes in the limit. Under this presupposition, we obtain the following from the residue theorem:

$$
j_{l}\left(k_{i}\left(\omega_{0}\right) r\right)=\sum_{p} \frac{U\left(\omega_{p}\right)}{U\left(\omega_{0}\right)} \frac{D^{+}\left(\omega_{0}\right)}{\partial_{\omega} D^{+}\left(\omega_{p}\right)} \frac{\exp \left[i k_{o}\left(\omega_{p}\right) a\right]}{\exp \left[i k_{o}\left(\omega_{0}\right) a\right]} \frac{j_{l}\left(k_{i}\left(\omega_{p}\right) r\right)}{\left(\omega_{0}-\omega_{p}\right)},
$$

where the sum runs over the poles of the eigenvalue equation $D^{+}=0$, and we assumed no other poles in $G(\omega)$ except at $\omega_{p}$ and $\omega_{0}$, with the latter giving rise to the left-hand side. The absence of additional poles requires that $U(\omega)$ has no poles except at $\omega=0$, and we will specify its behavior for small $\omega$ later. On inserting (21) into (18) and integrating over the real-frequency spectrum, we have

$$
E^{l, m}(r)=\sum_{q} C_{q}^{l, m} j_{l}\left(k_{i}\left(\omega_{q}\right) r\right),
$$

which can be read as an expansion of the inner-cavity field into a series in leaky modes.

For this strategy to work, we need to understand the analytic properties of our auxiliary $G$ and prove that the steps outlined can in fact be executed. In particular, we need to consider the role of possible branch cuts caused by $n(\omega)$, the behavior of $G$ at infinity, and the fact that solutions of the resonant eigenvalue equation exhibit three accumulation points. One is at infinity and the other two are located at $\omega=\omega_{3,4}$. To our best knowledge, these issues have not been considered together in detail in the previous treatments of normal-mode expansions in chromatically dispersive cavities.

First, let us think about the branch cuts inherited from the refractive index. Note that the "parity" of $D^{+}$in $n(\omega)$ is the same as that of $j_{l}$. This is why $G$ is even with respect to $n(\omega)$ and has no branch cut. Indeed, direct numerical inspection confirms that it varies smoothly as one crosses the line between $\omega_{3,4}$

The next important property is the behavior of $G$ at infinity. For very large $|\omega|$, all arguments appearing in the sphericals are also large, and we can utilize large-argument asymptotics of $j_{l}$ and $h_{l}^{1,2}$ to find the limiting form of $D^{+}$, namely,

$$
D^{+} \sim \frac{\exp \left[i k_{o} a\right]}{2 i^{l+1}}\left[-\cos \left[a k_{i}-\frac{l \pi}{2}\right]+\frac{i}{n} \sin \left[a k_{i}-\frac{l \pi}{2}\right]\right],
$$

and use it to estimate

$$
G(\omega) \sim \frac{U(\omega) \exp \left[i k_{o} a\right] j_{l}\left(k_{i} r\right)}{\frac{\exp \left[k_{o} a\right]}{2 i^{+}+1}\left(\omega_{0}-\omega\right)\left[-\cos \left[a k_{i}-\frac{l \pi}{2}\right]+\frac{i}{n} \sin \left[a k_{i}-\frac{l \pi}{2}\right]\right]} .
$$

At this point, the role of the exponential inserted into $G$ becomes obvious; it is meant to cancel the limiting form of the spherical Hankel in the denominator so that we obtain

$$
G(\omega) \sim \frac{2 i^{l+1} U(\omega)}{k_{i} r} \frac{\sin \left[k_{i} r-l \pi / 2\right]}{\left(\omega_{0}-\omega\right)\left[-\cos \left[a k_{i}-\frac{l \pi}{2}\right]+\frac{i}{n} \sin \left[a k_{i}-\frac{l \pi}{2}\right]\right]} .
$$


The large- $\omega$ behavior here is dictated by the trigonometric functions, in which $k_{i}(\omega), k_{0}(\omega) \rightarrow \omega / c$ and their arguments grow. Both, in the numerator and in the denominator, one of the exponentials contributing to the trig functions will dominate, and we obtain that as $|\omega| \rightarrow \infty$ the integrand becomes exponentially small,

$$
G(\omega) \sim \exp \left(\frac{|\operatorname{Im}\{\omega\}|}{c}(r-a)\right),
$$

as long as point $r$ lies inside the spherical cavity and $\operatorname{Im}\{\omega\}$ does not vanish.

The points at which the integration contours cross the real axis (i.e., $\operatorname{Im}\{\omega\}=0$ ) do not cause problems either. To see this, one has to know something that we will demonstrate later, namely, that the eigenvalues which accumulate around infinity appear far from the real axis. That is why the contribution from the integration contour in the vicinity of the real axis decays as $U(R) / R$. Thus, if our as yet free-to-choose $U(\omega)$ stays bounded at infinity, the integral along the big circle can be neglected for large $R$.

So far, our argument is at the same, in fact unsatisfactory, level as in some previous studies, where the mere fact that Green's function decays to zero for large $\omega$ is considered sufficient for the large (semi-)circle contour integral to vanish in the limit. However, the chromatic dispersion complicates the situation significantly because it introduces accumulation points for the complex resonance frequencies at $\omega_{3,4}$ and $\infty$.

It is not straightforward to verify that the special section of the integration contour can be neglected in the large- $R$ limit. So we first investigate the convergence of the leaky-mode representation (21). Only after we will have proven the convergence in Sec. V, we shall go on to show that the series in fact converges to the correct target.

\section{CONVERGENCE OF THE LEAKY-MODE EXPANSION}

The convergence properties of the leaky-mode expansion are controlled by the asymptotic behavior of poles in the vicinity of their accumulation points $\omega_{3,4}$ and $\infty$. We shall investigate the latter first.

\section{A. Complex eigenfrequencies and their accumulation point at $\infty$}

We require solutions of $D^{+}(\omega)=0$, in the vicinity of $\infty$, and they can be obtained with the help of the limiting form given in (23). While doing so, we also want to estimate $\partial_{\omega} D^{+}\left(\omega_{p}\right)$ and subsequently obtain the asymptotic behavior of the series (21).

For the location of pole $\omega_{p}$, labeled by an integer $p$, we have an equation

$$
\cos \left[a k_{i}\left(\omega_{p}\right)-\frac{l \pi}{2}\right]=\frac{i}{n\left(\omega_{p}\right)} \sin \left[a k_{i}\left(\omega_{p}\right)-\frac{l \pi}{2}\right]
$$

or equivalently

$$
\exp \left[2 i\left(a k_{i}\left(\omega_{p}\right)-\frac{l \pi}{2}\right)\right]=-\frac{n\left(\omega_{p}\right)+1}{n\left(\omega_{p}\right)-1} \sim \frac{2 \omega_{p}^{2}}{\omega_{p l}^{2}} .
$$

Large- $p$ poles can be found as $\omega_{p}=\omega_{p}^{\prime}+i \omega_{p}^{\prime \prime}$, where

$$
\omega_{p}^{\prime}=(2 p+l) \frac{\pi c}{2 a} \text { and } \omega_{p}^{\prime \prime}=-\frac{c}{2 a} \ln \left[\frac{2(p c \pi)^{2}}{4 a^{2} \omega_{p l}^{2}}\right] \sim-\frac{c}{a} \ln p .
$$

This result corroborates that the sequence of poles diverges away from the real axis as they approach infinity.

Now, we move to estimate a quantity that controls the convergence of the resonant-state expansion series,

$$
Q\left(\omega_{p}\right)=\frac{2 i^{l+1} U\left(\omega_{p}\right)}{k_{i} r\left(\omega_{0}-\omega_{p}\right)} \times\left.\frac{\sin \left[k_{i} r-l \pi / 2\right]}{\partial_{\omega}\left[-\cos \left[a k_{i}-\frac{l \pi}{2}\right]+\frac{i}{n} \sin \left[a k_{i}-\frac{l \pi}{2}\right]\right]}\right|_{\omega=\omega_{p}} .
$$


To evaluate the derivative term, one needs to realize that derivatives of the refractive index give negligible contributions because the index tends to unity for a large frequency. The dominant part of the above expression therefore becomes

$$
Q\left(\omega_{p}\right) \sim \frac{U\left(\omega_{p}\right)}{\omega_{p}^{2}} \frac{\sin \left[k_{i} r-l \pi / 2\right]}{\sin \left[a k_{i}-\frac{l \pi}{2}\right]+\frac{i}{n} \cos \left[a k_{i}-\frac{l \pi}{2}\right]} .
$$

On replacing cos with the help of the eigenvalue equation (27), we obtain

$$
Q\left(\omega_{p}\right) \sim \frac{U\left(\omega_{p}\right)}{\omega_{p}^{2}} \frac{\sin \left[k_{i} r-l \pi / 2\right]}{\sin \left[a k_{i}-\frac{l \pi}{2}\right]} \frac{\omega_{p}^{2}}{\omega_{p l}^{2}}
$$

and in terms of the pole index $p$, after using the asymptotic pole location (29),

$$
Q\left(\omega_{p}\right) \sim U\left(\omega_{p}\right) p^{\frac{r}{a}-1} .
$$

These results allow us to choose $U$ such that the series will converge. For example, for $U(\omega)=\omega^{-1}$, the series will converge for $r<a$, as can be verified by the direct comparison test and the integral test to show that the right-hand side is absolutely convergent. However, the rate of convergence becomes extremely slow as $r$ approaches the sphere radius at $r=a$. This is why one expects that the error in a truncated leaky-mode expansion of a function will be large in the vicinity of the cavity boundary.

\section{B. Complex eigenfrequencies and their accumulation at $\omega_{3}$}

Since the behavior in the vicinity of $\omega_{4}$ and $\omega_{3}$ is completely analogous due to symmetry, we shall only discuss the latter. Because in the neighborhood of $\omega_{3}$ the refractive index diverges, the arguments $k_{i} a$ found in the spherical Bessel functions are large, and one can replace these functions with their asymptotic expansions. This results in the eigenvalue equation given earlier,

$$
-\cos \left[a k_{i}\left(\omega_{p}\right)-\frac{l \pi}{2}\right] h_{l}^{(1)}\left(k_{o}\left(\omega_{p}\right) a\right)-\frac{1}{n} \sin \left[a k_{i}\left(\omega_{p}\right)-\frac{l \pi}{2}\right] h_{l+1}^{(1)}\left(k_{o}\left(\omega_{p}\right) a\right)=0 .
$$

One can verify that the solution of this equation that approaches $\omega_{3}$ as $p \rightarrow \infty$ is

$$
\omega_{p}-\omega_{3}=\frac{4 a^{2} \omega_{3}^{2}\left(\omega_{1}-\omega_{3}\right)\left(\omega_{2}-\omega_{3}\right)}{\pi^{2} c^{2}(1+l+2 p)^{2}\left(\omega_{3}-\omega_{4}\right)} .
$$

Next, we need to estimate the pole residues. The dominating part will arise from the derivative of the refractive index in the vicinity of $\omega_{3}$, so one only needs to consider the following terms:

$$
\left.\partial_{\omega} D^{+}\right|_{\omega \rightarrow \omega_{3}} \sim \frac{k_{o}^{2} a^{2}}{2} n^{\prime}(\omega)\left[\sin \left[a k_{i}-\frac{l \pi}{2}\right] h_{l}^{(1)}\left(k_{o} a\right)-\frac{1}{n} \cos \left[a k_{i}-\frac{l \pi}{2}\right] h_{l+1}^{(1)}\left(k_{o} a\right)\right] .
$$

Because the second term in square brackets is small and sin is close to unity, we obtain

$$
\left.\partial_{\omega} D^{+}\right|_{\omega \rightarrow \omega_{3}} \sim(-1)^{p} \frac{k_{o}^{2} a^{2}}{2} n^{\prime}(\omega) h_{l}^{(1)}\left(k_{o} a\right) \sim(-1)^{p} p^{3},
$$

where we have used $n^{\prime}\left(\omega_{p}\right) \sim p^{3}$. This implies that the series coefficient will scale with $p$ as

$$
Q\left(\omega_{p}\right) \sim(-1)^{p} \frac{j_{l}\left(k_{i} r\right)}{p^{3}} \sim(-1)^{p} \frac{\sin \left(k_{i} r-\frac{l \pi}{2}\right)}{k_{i} r p^{3}} \sim \frac{1}{p^{4}},
$$

which in turn indicates that the contribution of the leaky-mode series from modes with frequencies around $\omega_{3}$ converges rather rapidly.

The results of Subsection V A show that with a suitable choice of $U(\omega)$, the leaky-mode representation of the right-hand side in (21) does converge inside the spherical cavity. However, this fact alone does not guarantee that the convergence is to the desired target function that appears on the left in Eq. (21). To prove this last piece, we have to return to consider the special sections of the integration contour (see Fig. 1) and show that their contributions vanish in the limit. 


\section{Contribution of special sections of the integration contour: I}

Here we show that the integral

$$
I_{3}=\int_{C_{3}} G(\omega) d \omega
$$

vanishes in the limit of a small radius of $C_{3}$. Due to the symmetry, the argument will imply the same result for the integral along $C_{4}$.

In the vicinity of $\omega_{3}$, the behavior of function $G(\omega)$ becomes most influenced by the divergent refractive index which for $\omega \rightarrow \omega_{3}$ behaves as

$$
n(\omega) \approx \frac{n_{3}}{\sqrt{\omega-\omega_{3}}} \text { with } n_{3} \equiv \sqrt{\left(\omega_{3}-\omega_{1}\right)\left(\omega_{3}-\omega_{2}\right) /\left(\omega_{3}-\omega_{4}\right)} .
$$

With this, the leading term in $G$ for $\omega \rightarrow \omega_{3}$ can be written as

$$
G(\omega) \approx \sqrt{\omega-\omega_{3}} \frac{\sin \left(\frac{n_{3} \omega_{3} r}{\sqrt{\omega-\omega_{3}}}-\frac{l \pi}{2}\right)}{\cos \left(\frac{n_{3} \omega_{3}}{\sqrt{\omega-\omega_{3}}}-\frac{l \pi}{2}\right)},
$$

where we have left out constant factors that are irrelevant for our considerations. We shall find a bound for this function valid in the limit of shrinking $C_{3}$ and utilize it to show that the integral vanishes.

Let us parameterize contour $C_{3}$ through angle $\theta$,

$$
\omega=\omega_{3}+\xi_{p} e^{\theta} \text { with } \omega_{3}=\left|\omega_{3}\right| e^{i \phi_{3}},
$$

using $\xi_{p}$ standing for the radius of the circle. We will select a sequence of diminishing radii, labeled by integer $p$,

$$
\xi_{p}=\left(\frac{n_{3}\left|\omega_{3}\right|}{\pi(p+l / 2)}\right)^{2} \sim p^{-2}, p \rightarrow \infty .
$$

Note that $\xi_{p}$ is chosen to avoid the leaky-mode poles. More precisely, the integration path travels through the midpoint between a pair of poles given by (35). On inserting our parameterization into (41), we obtain the integrand as a function of $\theta$ and must distinguish two cases, namely, $\theta / 2$ equal to and/or different from $\phi_{3}$.

For small $\xi_{p}$ and $\theta / 2 \neq \phi_{3}$, we find

$$
G\left(\xi_{p}, \theta\right) \sim e^{i \theta / 2} \sqrt{\xi_{p}} \exp \left[\frac{n_{3}(r-1)\left|\omega_{3}\right|\left|\sin \left(\phi_{3}-\theta / 2\right)\right|}{\sqrt{\xi_{p}}}\right] .
$$

In other words, as long as away from the special $\theta / 2=\phi_{3}$, and for $r<1, G(\theta)$ is exponentially small when $p \rightarrow \infty$.

At the special point on the contour where $\theta / 2=\phi_{3}$, we evaluate

$$
G\left(\xi_{p}, \theta / 2=\phi_{3}\right) \sim \frac{(-1)^{p}}{(2 p+l)} \sin \left(\frac{\pi}{2}(l-l r-2 p r)\right) .
$$

Results (44) and (45) show that a global bound for the integrand $G$ on $C_{2}$ scales as $p^{-1}$. From (43) we know that the circumference of the circle scales as $p^{-2}$, and thus the integral $I_{3}$ approaches zero at least as fast as $p^{-3}$,

$$
I_{3} \sim p^{-3} \rightarrow 0
$$

\section{Contribution of special sections of the integration contour: II}

In this section, the goal is to show that the integral along a large-radius circle $C_{p}$

$$
I_{p}=\int_{C_{p}} G(\omega) d \omega
$$


vanishes in the limit of large index $p$. Because of the symmetry of the pole positions, we only need to consider the part of the sequence of growing circular contours $C_{p}$ that are located in the right half-plane. These parts, denoted by $C_{p}^{+}$, are parameterized using

$$
\omega(\theta)=\left|\bar{\omega}_{p}\right| e^{i \theta}, \quad-\frac{\pi}{2}<\theta<\frac{\pi}{2},
$$

where $\bar{\omega}_{p}=\frac{1}{2}\left(\omega_{p+1}+\omega_{p}\right)$ denotes the midpoint between consecutive poles in the sequence of poles (29) approaching infinity. Using standard asymptotic formulas for the spherical Bessel and Hankel functions, as we have done several times before in this paper, we find the following bound for $G(\omega)$ :

$$
\left|G\left(\left|\bar{\omega}_{p}\right| e^{i \theta}\right)\right| \lesssim U\left(\left|\bar{\omega}_{p}\right|\right) e^{-(R-r)\left|\bar{\omega}_{p}\right| \sin (|\theta|)},
$$

where we disregard any $p$ independent constants. The bound (49) is derived under the assumption that the angle $\theta$ is different from zero and the argument of the midpoint position, $\left|\bar{\omega}_{p}\right|$. However, we find through detailed analysis that the bound also holds at these two special angles.

Using the global bound (49) we have

$$
\begin{aligned}
\left|\int_{C_{p}^{+}} G(\omega) d l\right| \lesssim\left|\bar{\omega}_{p}\right| U\left(\left|\bar{\omega}_{p}\right|\right) \int_{-\frac{\pi}{2}}^{\frac{\pi}{2}} e^{-(R-r)\left|\bar{\omega}_{p}\right| \sin (|\theta|)} d \theta \\
\sim \frac{1}{R-r} U\left(\left|\bar{\omega}_{p}\right|\right),
\end{aligned}
$$

from which we can conclude that the contribution from the circle $C_{p}^{+}$and thus by symmetry the whole circle $C_{p}$ vanish in the limit when $p$ approaches infinity if we chose $U(\omega)=\omega^{-1}$.

Thus, from Secs. IV and V we can conclude that for the choice $U(\omega)=\omega^{-1}$, not only does the leaky-mode series converge inside the dielectric sphere, but it also converges to the right function.

\section{OVERCOMPLETENESS RELATIONS}

It is well known that the leaky-mode functions are in general not linearly independent and are subject to various over-completeness relations. Our method for construction of the leaky-mode expansion for the electromagnetic field inside the cavity can be easily adopted for the derivation of such relations. This is the subject of Sec. VII.

The method outlined in Sec. IV highlights the fact that there is a degree of freedom embodied in the function $U(\omega)$. So far, we have only utilized this to fulfill conditions necessary for the leaky-mode expansion convergence. In particular, $U(\omega)$ must not have a pole higher than $1 / \omega^{l-1}$ at the origin. This is to avoid creation of a new pole of $G(\omega)$ at zero. Because the behavior of the spherical Bessel function for small arguments is such that $j_{l}\left(k_{i}(\omega) r\right) \sim \omega^{l}$, it follows that $U \sim 1 / \omega^{l-1}$ will not cause appearance of a pole in $G(\omega)$ at the origin of the $\omega$-plane. Also, for the series to converge, $U$ has to decay at least as $1 / \omega$ at large frequencies, otherwise the line-integral $\int_{C} G(\omega) d \omega$ contribution from the big circle $C$ would not tend to zero as its radius increases.

Naturally, a faster decrease would not interfere with the convergence, which can be easily verified by revisiting our arguments. For the contribution to the series originating in the accumulation point $\omega_{3}$, our "gauge" function $U(\omega)$ behaves essentially as a constant and changes nothing essential on the asymptotic behavior of the expansion terms. In the neighborhood of the infinity, faster decay of $U$ can only improve the rate of convergence since it further suppresses the contribution from the contour integral.

Bearing these properties in mind, we take the expansion (21) in the limit $\omega_{0} \rightarrow 0$,

$$
\left(n(0) \omega_{0} r\right)^{l}=\sum_{p} \frac{U\left(\omega_{p}\right)}{U\left(\omega_{0}\right)} \frac{D^{+}(0)}{\partial_{\omega} D^{+}\left(\omega_{p}\right)} \frac{\exp \left[i k_{o}\left(\omega_{p}\right)\right]}{1} \frac{j_{l}\left(k_{i}\left(\omega_{p}\right) r\right)}{\left(\omega_{p}\right)},
$$

and choose

$$
U(\omega)=\omega^{-k}
$$

to obtain

$$
\sum_{p} \frac{\exp \left[i k_{o}\left(\omega_{p}\right)\right] j_{l}\left(k_{i}\left(\omega_{p}\right) r\right)}{\partial_{\omega} D^{+}\left(\omega_{p}\right)\left(\omega_{p}\right)^{k+1}}=0 .
$$


For the allowed values of $k \in 1,2, \ldots, l-1$, these formulas represent the over-completeness relations. It is not clear that these are all possible over-completeness relation or if an arbitrary overcompleteness relation can be expressed as a linear combination of those described above.

However, the existence of these relations means that the leaky-mode expansion is not unique. This is not to say that the resonant-expansion sum would converge to different results-if the series converges, it tends to the unique function given by the physical initial value problem. Non-uniqueness only means that there are different series converging to the same target. The fact that the leaky modes are linearly dependent indicates that for almost any choice of $U$, significant cancellations must occur in the series, and this is yet another reason why one should not expect an easy-to-evaluate (from the numerical standpoint) expansion. On a positive side, this might also open the possibility to speed-up the convergence by adding to the given series a "suitable" combination of leaky modes that add up to zero.

\section{SUMMARY AND CONCLUDING REMARKS}

We have studied the mathematical properties of leaky-mode expansions for an electromagnetic field excited inside a spherical cavity made of dispersive dielectric. Our investigation into the convergence properties of the leaky-mode series is the first to take into account a truly realistic model of the medium which is based on the Lorentz oscillator model without any dispersion-less background. In other words, our results apply to an arbitrary frequency range and reflect the fact that high-frequency waves must experience vanishingly small contrast between the inner medium and the outside vacuum.

This work provides a rigorous mathematical justification for a recently proposed method to construct the leaky-mode expansions and to generate the so-called over-completeness relations. Note that our approach differs from the usual treatment based on Green's functions which assume that the asymptotic part of the expanded electromagnetic field only consists of outgoing waves. By contrast, our formulation concerns the arbitrary electromagnetic field excited inside the cavity and does not rely on the boundary conditions at infinity. In effect, our method is to expand, directly, the realfrequency spherical Bessel functions that form the continuum basis for the electromagnetic fields in the spherical cavity. While the new approach was justified here for the case of a homogeneous spherical cavity, we trust that a generalization for more complex systems, in particular those with spherical symmetry, should be possible.

We have also studied the rate of the convergence of the resulting series and showed how this depends on the position inside the sphere. Typically, the first few terms of the leaky-mode series result in quite good approximations (see, e.g., illustrations in Ref. 31), but we have demonstrated here that the eventual convergence may be exceedingly slow and decelerates even more for locations closer to the material interface. Our convergence-rate results help us to understand this behavior, which we have identified as originating from the existence of the resonant-pole accumulation point at infinity.

Besides the infinity, the dispersive medium gives rise to additional accumulation points of complex energies of leaky modes. They originate from the singularities of the refractive index as modeled by the Lorentz oscillator. For a rigorous completeness argument, such points must be excised from the region enclosed within the integration contour used to convert the continuum expansion into a discrete series. Here we have shown that these additional accumulation points do not affect the rate of convergence of the leaky-mode series.

It should be noted that the accumulation points arise naturally in dispersive media and are therefore present also in what is a more standard treatment based on Green's functions. While in the light of our observation it is not likely that previous results should be affected, these issues have not yet been discussed within Green's function methods dealing with three-dimensional dispersive cavities.

The way we construct the leaky-mode expansions and their over-completeness relations makes it obvious that the representation is not unique for a given function. Because the leaky modes are not linearly independent, cancellations must occur during the series summation, and we feel that these may contribute to the poor asymptotic convergence of the series. Looking ahead, it would be 
interesting to identify all possible over-completeness relations and utilize them to eliminate such cancellations in an attempt to achieve a faster convergence.

\section{ACKNOWLEDGMENTS}

This material is based upon work supported by the Air Force Office of Scientific Research under Award No. FA9550-13-1-0228.

${ }^{1}$ N. Moiseyev, “Quantum theory of resonances: Calculating energies, widths and cross-sections by complex scaling," Phys. Rep. 302(5-6), 212-293 (1998).

${ }^{2}$ E. S. C. Ching, P. T. Leung, A. Maassen van den Brink, W. M. Suen, S. S. Tong, and K. Young, "Quasinormal-mode expansion for waves in open systems," Rev. Mod. Phys. 70, 1545-1554 (1998).

${ }^{3}$ E. F. Franchimon, K. R. Hiremath, R. Stoffer, and M. Hammer, "Interaction of whispering gallery modes in integrated optical microring or microdisk circuits: Hybrid coupled mode theory model," J. Opt. Soc. Am. B 30(4), 1048-1057 (2013).

${ }^{4}$ J. Rosenkrantz de Lasson, P. T. Kristensen, J. Mørk, and N. Gregersen, "Roundtrip matrix method for calculating the leaky resonant modes of open nanophotonic structures," J. Opt. Soc. Am. A 31(10), 2142-2151 (2014).

${ }^{5}$ P. T. Kristensen and S. Hughes, "Modes and mode volumes of leaky optical cavities and plasmonic nanoresonators," ACS Photonics 1(1), 2-10 (2014).

${ }^{6}$ A. Settimi, Classical and Quantum Approach of Quasi Normal Modes in Linear Regime: An Application to One Dimensional Photonic Crystals (LAP LAMBERT Academic Publishing, 2010).

${ }^{7}$ A. Settimi, S. Severini, and B. J. Hoenders, "Quasi-normal-modes description of transmission properties for photonic bandgap structures,” J. Opt. Soc. Am. B 26(4), 876-891 (2009).

${ }^{8}$ R.-C. Ge, J. F. Young, and S. Hughes, "Quasi-normal mode approach to the local-field problem in quantum optics," Optica 2(3), 246-249 (2015).

${ }^{9}$ F. Yang, H. Liu, H. Jia, and Y. Zhong, "Analytical description of quasi-normal mode in resonant plasmonic nano cavities," J. Opt. 18(3), 035003 (2016).

${ }^{10}$ P. T. Leung, Y. T. Liu, W. M. Suen, C. Y. Tam, and K. Young, "Perturbative approach to the quasinormal modes of dirty black holes," Phys. Rev. D 59, 044034 (1999).

${ }^{11}$ B. Vial, F. Zolla, A. Nicolet, and M. Commandré, "Quasimodal expansion of electromagnetic fields in open two-dimensional structures," Phys. Rev. A 89, 023829 (2014).

${ }^{12}$ M. Shlafman, I. Bayn, and J. Salzman, "Effect of dielectric constant tuning on a photonic cavity frequency and q-factor," Opt. Express 18(15), 15907-15916 (2010).

${ }^{13}$ S.-Y. Lee, "Decaying and growing eigenmodes in open quantum systems: Biorthogonality and the Petermann factor," Phys. Rev. A 80, 042104 (2009).

${ }^{14}$ P. T. Leung, S. S. Tong, and K. Young, "Two-component eigenfunction expansion for open systems described by the wave equation I: Completeness of expansion,” J. Phys. A: Math. Gen. 30(6), 2139 (1997).

${ }^{15}$ A. M. van den Brink, "Exactly solvable path integral for open cavities in terms of quasinormal modes," Phys. Rev. E 61, 2367-2375 (2000).

${ }^{16}$ A. M. van den Brink and K. Young, "Jordan blocks and generalized bi-orthogonal bases: Realizations in open wave systems," J. Phys. A: Math. Gen. 34(12), 2607 (2001).

${ }^{17}$ P. T. Leung, K. M. Pang, and K. Young, "Two-component wave formalism in spherical open systems," J. Phys. A: Math. Gen. 39(1), 247 (2006)

${ }^{18}$ A. Settimi and S. Severini, "Linking quasi-normal and natural modes of an open cavity,” J. Mod. Opt. 57(16), 1513-1525 (2010).

${ }^{19}$ E. A. Muljarov and W. Langbein, "Exact mode volume and Purcell factor of open optical systems," Phys. Rev. B 94, 235438 (2016)

${ }^{20}$ M. B. Doost, W. Langbein, and E. A. Muljarov, "Resonant-state expansion applied to planar open optical systems," Phys. Rev. A 85, 023835 (2012).

${ }^{21}$ M. B. Doost, W. Langbein, and E. A. Muljarov, "Resonant state expansion applied to two-dimensional open optical systems," Phys. Rev. A 87, 043827 (2013).

${ }^{22}$ M. B. Doost, W. Langbein, and E. A. Muljarov, "Resonant-state expansion applied to three-dimensional open optical systems," Phys. Rev. A 90, 013834 (2014).

${ }^{23}$ N. Moiseyev, Non-Hermitian Quantum Mechanics (Cambridge University Press, 2011).

${ }^{24}$ W. Broer and B. J. Hoenders, "Natural modes and resonances in a dispersive stratified n -layer medium,” J. Phys. A: Math. Theor. 42(24), 245207 (2009).

${ }^{25}$ S. M. Dutra and G. Nienhuis, "Quantized mode of a leaky cavity," Phys. Rev. A 62, 063805 (2000).

${ }^{26}$ P. T. Kristensen, C. Van Vlack, and S. Hughes, "Generalized effective mode volume for leaky optical cavities," Opt. Lett. 37(10), 1649-1651 (2012).

${ }^{27}$ P. T. Kristensen, R.-C. Ge, and S. Hughes, "Normalization of quasinormal modes in leaky optical cavities and plasmonic resonators," Phys. Rev. A 92, 053810 (2015).

${ }^{28}$ W. Yu, W. Yue, P. Yao, Y. Lu, and W. Liu, “Approach to calculate normal modes by decomposing the dyadic Green's function,” Opt. Express 22(22), 26712-26719 (2014).

${ }^{29}$ P. T. Leung, S. Y. Liu, and K. Young, "Completeness and time-independent perturbation of the quasinormal modes of an absorptive and leaky cavity,” Phys. Rev. A 49, 3982-3989 (1994).

${ }^{30}$ P. T. Leung, S. Y. Liu, and K. Young, "Completeness and orthogonality of quasinormal modes in leaky optical cavities," Phys. Rev. A 49, 3057-3067 (1994). 
${ }^{31}$ M. Mansuripur, M. Kolesik, and P. Jakobsen, "Leaky modes of dielectric cavities," Proc. SPIE 9931, 99310B (2016).

${ }^{32}$ M. Mansuripur, M. Kolesik, and P. Jakobsen, "Leaky modes of solid dielectric spheres," Phys. Rev. A 96, 013846 (2017).

${ }^{33}$ Z. Hradil, "Decomposition of the electromagnetic field in lossless inhomogeneous dispersive dielectrics," Phys. Rev. A $\mathbf{5 3}$ 3687-3690 (1996).

${ }^{34} \mathrm{Z}$. Li, G. Yi-Bo, and W. Cheng, "Green function and perturbation method for dissipative systems based on biorthogonal basis," Commun. Theor. Phys. 51(6), 1017 (2009). 